\title{
The habituation of forearm muscle blood flow in phobic subjects
}

\author{
HARRY BRIERLEY
}

From the Professorial Unit of Psychological Medicine, General Hospital, Newcastle upon Tyne

In recent years, renewed attention has been paid to the possible contribution which physiological measurements might make to the understanding and treatment of neuroses. In particular, the studies by Lader and Montagu (1962) of the psychogalvanic reflex, and by Harper, Gurney, Savage, and Roth (1965) and Kelly (1966) of muscle blood flow, excite new interest in techniques of this class. The measurement of anxiety under stress (Vanderhoof and Clancy, 1962) and the observation of the influence of leucotomy (Kelly, Walter, and Sargant, 1966), psychotherapy (Vanderhoof and Clancy, 1964), or drugs (Lader, 1964; Lader and Wing, 1965), are important steps forward. The results appear most promising in that clear normal/neurotic differences are regularly found. The most important researches would appear to be those of Lader and Kelly following the separate tracks of psychogalvanic reflex (PGR) and muscle blood flow. It was in the hope of drawing together these fields, somewhat, that the present investigation was undertaken.

Lader (1964) found that subjects reacted to a mild stressor, an intermittent sound, with an increase in PGR. This response habituated with repetition of the stressor but normal subjects differed from anxious subjects in their rates of habituation. This led Lader to speculate on the role of stress-habituation in the formation of phobic symptomatology. Perhaps phobic panic attacks might arise from a positive feedback process. That is to say, a normal person reentering a stress situation will adapt or habituate in his arousal response, anxiety diminishing with each re-entry. However, if the rate of habituation was small or indeed negative, an accumulative response would occur, the anxiety response aroused by the new entry would be compounded with the unduly persisting response from previous experiences. The effect would be to produce rapidly incubating arousal in the situation out of proportion to its apparent anxiety provoking values.

Harper et al. (1965) and Kelly (1966) both found that the response to a stress situation was reflected in muscle blood flow. However, neither found that the degree of response was greater in the anxiety states than in normal individuals. Kelly (1967) has reported that phobic patients are not differentiated from normals in resting blood flow either, although Marks (to be published) suggests that the monosymptomatic phobias show relatively normal blood flows whereas the agoraphobias yield rather higher values. On the other hand, the role of muscle blood flow in syncopal attacks-for example, Barcroft and Edholm (1945) - would seem highly relevant to phobic symptomatology. Roth (1960) draws attention to the common occurrence of syncopal attacks (as well as less well-defined sensations of giddiness) in patients with phobic anxiety states. Lader (1964) reports also the case of an anxious patient who showed normal psychogalvanic reflex responses which coincided with depersonalization experiences. It seemed possible that the symptom had a normalizing effect and might be viewed as a mechanism with a protective role against excessive autonomic arousal.

Unlike studies of PGR, those of blood flow are at a disadvantage. They require cumbersome apparatus; most available methods of measurement require a mechanical constriction of the arm which itself is a not unaversive stressor for many people; and continuous readings cannot be made.

The method generally employed-for example, by Kelly-is that of Barcroft (Barcroft and Swan, 1953). It requires the forearm to be immobilized in a temperature-controlled water jacket. Studies have invariably been carried out with the patient close by the measuring apparatus and measurements have even been made while the patient is in conversation with the investigator.

The experimental stressors employed in the blood flow studies differ from the controlled sound stressor used in Lader's PGR studies. Mental arithmetic is frequently used as a stressor and is regarded, by implication, as anxiety producing.

However, Mathews (to be published) found that mental arithmetic seems capable of producing greater increase in blood flow than imagery of situations producing phobic fears. Moreover, mental arithmetic, although producing an arousal response, 
did not necessarily produce any response which could be readily identified as anxiety.

Arithmetic is a stressor which would appear likely to produce widely varying emotional responses from normal subjects. Certainly arithmetic has a profound defect as a stressor in that it is difficult to hold constant during periods of measurement which may be of the order of one minute. Moreover, it seems customary to use 'harassing comments' to confuse the picture even further, but Mathews attributes blood flow increases to 'arousal' rather than 'anxiety'. In any event, arithmetic seems to be a poorly controlled stressor, effective to a rather unexplained and unexpected degree in raising blood flow.

A further complication arises in the measurement of blood flow because of considerable variation in individual readings. Kelly (1967) discusses this problem and feels that an assessment of 'basal' relaxing blood flow is best obtained by taking the mean of the three lowest readings. While the method assumes that greatest relaxation and lowest blood flow are coincident, it has the serious risk of selecting readings with maximum experimental error. It also neglects the fact that the general level of blood flow may vary meaningfully throughout a session. Kelly (1967) has drawn attention to these variations as objections to the use of mean measurement values, but his solution is rather arbitrary. Lader's findings on PGR habituation which one would expect to replicate in blood flow studies could not of course be examined by Kelly's method of measurement of 'basal' flow rates.

\section{METHODS}

The study of stress response in phobic patients is especially important in that it may lead to objective methods of identifying stressors and monitoring therapeutic methods such as desensitization and re-training regimes. Workers who have investigated muscle blood flow of patients under stress have found disappointing results, the responses of normal and phobic patients being similar; however, as discussed above, the techniques and methods of measurement might be at fault to some degree. The present investigation sought to modify the method of investigation in three ways: (1) use of the strain gauge plethysmograph; (2) reduction of distracting stimuli and an improvement in relaxation conditions; (3) use of a more controlled stressor.

The strain gauge plethysmograph has been fully described by Whitney (1953). The only modification to this procedure was to adopt a transistorized bridge circuit and amplifier, records being made on a recording microammeter. This apparatus was in fact a development of that used by Harper et al. (1965).

The method employs a mercury in rubber strain gauge in place of the cumbersome water jacket. This gauge is $0.5 \mathrm{~mm}$ bore silicone rubber tube attached to a clip which incorporates a calibrating adjustment. The tube is looped around the forearm quite comfortably and is linked with the measuring apparatus by hearing-aid flex. Thus the patient is barely aware of the presence of this part of the apparatus. Pressure cuffs are used as in the Barcrof equipment. The expansion of the limb circumferentiall causes a change in the electrical resistance of the stretche mercury column in the tube and this resistance change $\frac{18}{8 .}$ recorded on the pen recorder.

As Kelly (1967) points out, the strain gauge method is.a somewhat 'indirect' method, calculating volume changes' from girth changes, involving assumptions about the approximate circularity of the arm section. However, the results of the two methods show a very high intercorre lation. Whitney (1953) reports correlation of 0.80 to 0.95 between gauge values and the Barcroft plethysmograph values with the methods used simultaneously. The re-test reliability of the Barcroft method is of the order 0.9 also (Kelly, 1966). The absolute values obtained tend to differ somewhat by a constant factor apparently partly dependent on the method of gauge calibration and varying from about $10 \%$ (Whitney, 1963) to $25 \%$ (Whitney, 1953. Kelly's inference that the strain gauge is liable to mech anical breakdown is unmerited. In no case in the present experiment did any sort of breakdown occur. In comparis son, the fitting of the strain gauge is literally a 10-second matter and it in no way restricts the patient. This contrasts sharply with the 15 minutes required for settingoug the Barcroft apparatus (Kelly, 1966).

Given the high relationship between results of the \$w methods, absolute values are not important where groups are compared by one method or the other. Consequent in this investigation the over-riding consideration wasthes minimal interference with the subject. Additionally, the gauge method much simplified remote recordings. 蛙e patient was seated in an armchair specially padded thick polystyrene foam. Some experiments produced $\stackrel{Q}{Q}$ semi-reclining position where pads could be placed underp the polystyrene at each side of the head, under the lowe part of the back, and beneath the knees to support the patient somewhat. This proved a very comfortable pose tion, and, although patients could have moved freel and indeed were not instructed otherwise, none did so to any extent. The patient's right arm was used, supported on an adjustable rest.

The patient was seated in a semi-soundproofed room dimly illuminated. The cuffs were controlled by reservoir inflated from a compressed air cylinder and all controls: and recording apparatus were situated in an adjoining room. The process was described to the patients, especis ally how they would feel the cuffs inflate and deflate. The were instructed to relax and sleep if possible, though now did so. They were also warned that as it was necessary to have them awake at various stages they would be aroused by a noise from a set of loudspeakers in the room. This stressor was relayed by three speakers from a tape recorder. The noise itself was the much amplified tick of clock at a sound level of 80 to 85 decibels.

For each subject, readings were taken each 90 seconds Fifteen seconds were allowed for normalizing of the blood flow after the inflation of the distal cuff before inflating the proximal cuff (Kerslake, 1949). Thirty seconds waf 
the minimum time allowed between readings and, during this period, both cuffs were deflated. Other workers have kept the distal cuff inflated throughout periods of, say, 10 minutes. Except for the extra consumption of recording time, there appeared to be no advantages to offset the extra discomfort of the complete occlusion of the hand for long periods, and the present method was decided upon.

Each recording session began with five relaxed recordings. From then on, the first of each set of three recordings was taken while the noise stressor was being applied. This schedule gave five stressed recordings at intervals of four and a half minutes with 15 relaxing recordings. The period of examination was thus $\mathbf{3 0}$ minutes in all.

\section{SUBJECTS}

The experimental subjects were 10 cases of phobic anxiety in each of which agoraphobia predominated. None of the patients would be classed as monosymptomatic phobias. In the phobic group eight patients were female and two male, their ages ranging from 26 to 45 .

The normal subjects were drawn from hospital staff with no known psychiatric history. This group included seven females and three males. Their ages ranged from 21 to 40 , with a mean of $27 \cdot 1$ years as compared with $37 \cdot 8$ years with the phobic group. There was no evidence of a relationship between age and blood flow in these groups $(\rho=-0.19$ for neurotic groups; $\rho=+0.15$ normal groups).

While patients were helpful and co-operative in what they understood to be a research project, a few declined to take part on account of various pathological fears they outlined. At the same time ethical considerations were taken into account in limiting the size of the groups. No patient was gravely disturbed by the experiment, but a number were distinctly relieved when it was over.

\section{RESULTS}

The relationship between relaxed blood flow values and time was examined (Lader has used response number rather than time). As shown in Table I there was a rather better fit of mean values of blood flow for both groups on the regression lines on log time than on those with simple raw time values. The regression coefficients for both groups relaxing derived from the log time values were significant beyond the 0.01 level. The regression coefficient for phobics on time value was also significant at the 0.01 level but for the normal group the level of signifi-

TABLE I

FIT OF TIME AND LOG TIME REGRESSION LINES

\begin{tabular}{lcc}
\hline Group & \multicolumn{2}{c}{ Sample standard deviation from regression } \\
\cline { 2 - 3 } & Time regression line & Log time regression line \\
\hline Normals & 0.39 & 0.31 \\
Phobics & 0.19 & 0.15 \\
\hline
\end{tabular}

cance was only $0 \cdot 05$. Log time values were, therefore, accepted as giving a better approximation to a linear relationship, as was the case in Lader's PGR investigation. It is also clear that during a $\mathbf{3 0}$ minute period of relaxation there is an appreciable fall in muscle blood flow in both phobic and normal groups. Figure 1 shows the blood flow/log time regression lines for both relaxed and stressed values.

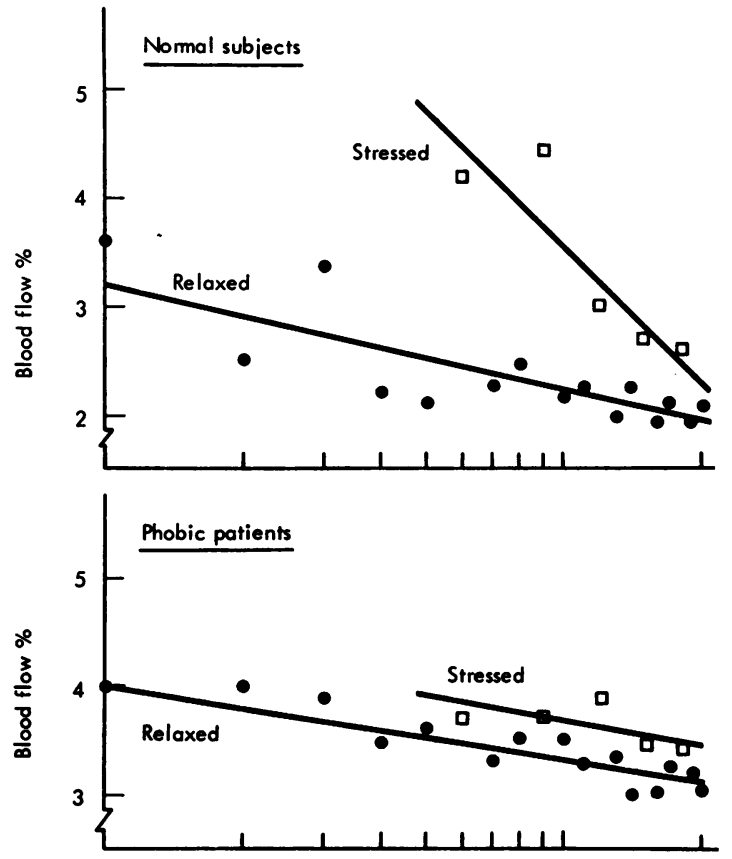

Log time units

FIG. 1. Habituation curves of blood flow.

Regression coefficients were calculated for each individual so that a comparison might be made between rates of blood flow habituation. The ' $b$ ' regression coefficient representing the slope of the

TABLE II

SIGNIFICANCE OF DIFFERENCES BETWEEN HABITUATION RATES-' $b$ ' REGRESSION COEFFICIENTS

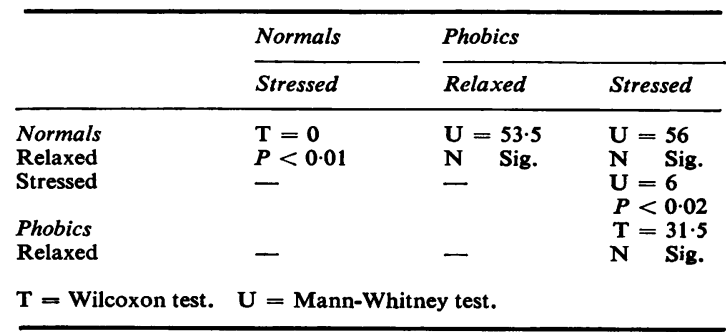


line can be taken as an index of rate of habituation. A comparison of the ' $b$ ' values appears in Table II. Non-parametric statistics are employed here because of the considerable departure from normality exhibited by the distributions of data. The 'a' regression constant is the value of blood flow at the intersection of the curve on that axis. It might be taken as indicating the general level of initial blood flow in the case of the relaxing curves. The ' $a$ ' values for the stressed responses are more ambiguous, representing a value depending directly upon both rate of habituation and the level of initial stressed response. They are quoted here only as a complex constant contrasting the two groups. A more meaningful point on the curve has been designated the ' 6 th value' level and is the theoretically predicted value, from each subject's stressed habituation curve, of the true level of blood flow at the first stressed recording. A comparison of ' $a$ ' and '6th values' appears in Table III.

The following four regression equations are those of the habituation curves of Fig. 1.

Normals relaxing $-y=-1.04 x+3.24$

Phobics relaxing $-\mathrm{y}=-0.66 \mathrm{x}+3.98$

Normals stressed $-y=-4.07 x+7.66$

Phobics stressed $-\mathrm{y}=-0.77 \mathrm{x}+4.42$

These equations are of the form $y=b x+a$, where $y$ is blood flow and $x \log$ time, and $b$ and $a$ have the meaning described above.

The graph can, therefore, be said to have the following principal features. The regression coefficients being statistically significant, habituation does clearly occur in both phobics and normals when relaxing. The fall in blood flow with time is very similar in magnitude, in both groups. However, the relaxing blood flow of these agoraphobic patients is higher than that of the normal controls. The rates of relaxation being almost identical, this can be taken as remaining so throughout the experiment. Under conditions of stress, the significantly different ' $a$ ' values indicate considerably different habituation curves and the difference in slope or rate of habituz tion is also significant. In fact, it is shown that the phobic group barely responds to the stressor, thex, is no change in the habituation rate and the bloof flow increase is well below statistical significance. If contrast, the normal group shows a large and higher significant increase in blood flow ('6th values') an this is accompanied by rapid habituation.

The relaxed and stressed habituation curves of the phobic group commence between the two normat curves but cross the normal stressed curve. This means that the difference between the phobic and normal 6th values is not quite large enough to reac? the 0.05 level of significance. However, the difference in the percentage increase in flow due to stress between the two groups is large.

The stressed response lines in this graph are strikingly similar to those obtained by Lader (1964) and, while little weight can be placed on the observa tion since neither the neurotic anxiety levels n@ stressors were equated, it should be recorded that the ratio of normal/neurotic habituation rates found Lader's research is 0.59 while the same ratio here tis 0.53 .

\section{DISCUSSION}

The principal result of this research is the strikfing agreement between the habituation findings from forearm muscle blood flow and those of PGR of tained by Lader. It confirms also the findings of ow writers that muscle blood flow is raised in patit exhibiting high anxiety. The findings concerning the responses to a stressor are less unequivocal. It is apparent that the stressor used changed the blood flo of the phobic patients to only a small degree, conz pared with the high response and rapid habituation of the normal group.

TABLE III

SIGNIFICANCE OF DIFFERENCES IN BLOOD FLOW VALUES

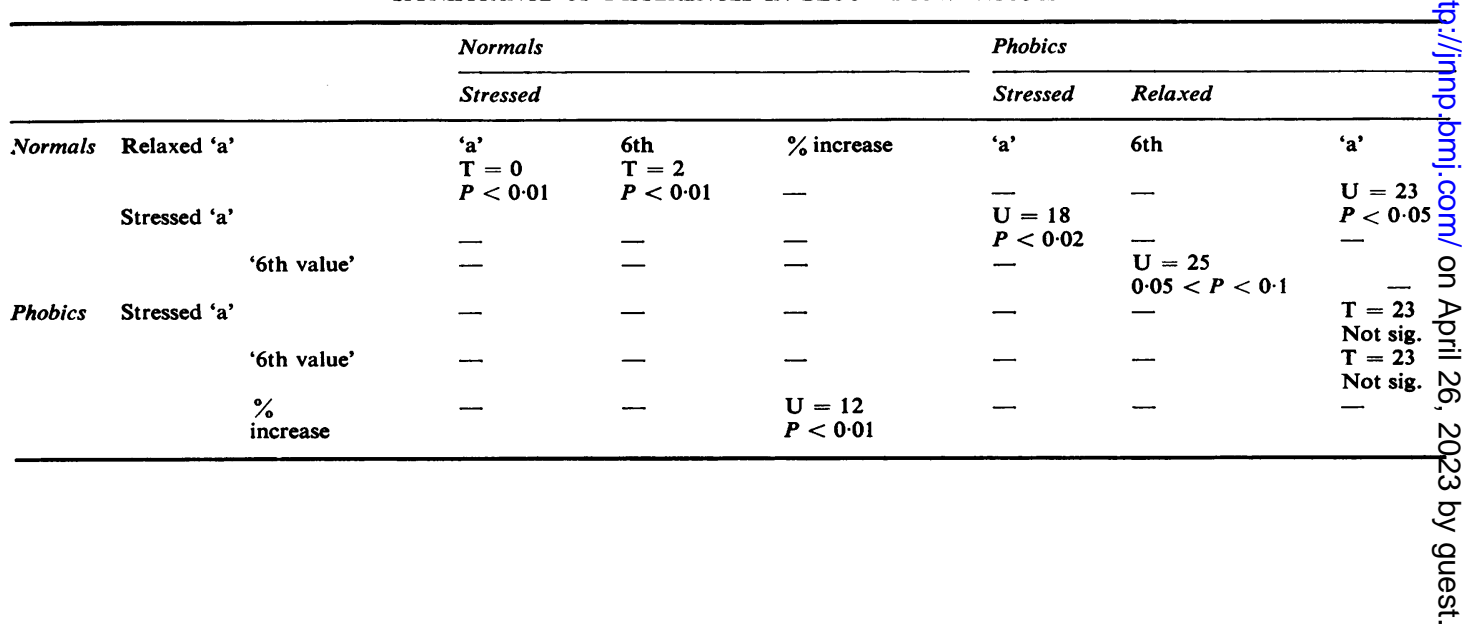


The phobic patients show stress response and relaxation response curves similar to the normal relaxation curve but at a higher level of blood flow. This amounts to a consistently enhanced level of arousal such as might be associated with a free floating anxiety level, so that at any stage of relaxation there is likely to be a constant difference in arousal between normal and phobic patients.

Kelly and Walter (1968) comment that patients with high levels of free floating anxiety are seldom if ever able to relax. The results here qualify this statement in the sense that relaxation takes place to a similar degree to the normal group but that a constant difference is maintained which represents a measure of the undiminishing 'free floating' anxiety.

Perhaps this observation suggests research into the separate evaluation of 'free floating' and specific anxiety reactions. The present agoraphobic patients are likely, for example, to be distinguished from those with monosymptomatic phobias in this way, and this could well be an explanation of the relative failure of desensitization methods in treating agoraphobias.

These results do not demonstrate the 'abnormal ease of arousal' in phobic states described by Kelly and Walter (1968). The experimental stress response among phobic patients is much lower than that of normal controls and not different from phobic relaxed curves, although the writer has certainly observed that much larger responses can be triggered off in agoraphobics by methods such as intensive fantasies of specific phobic situations. This conflicts with the explanation of the lack of experimental responses as a result of the persistent arousal in anxious patients taking up the 'capacity to react' (Kelly and Walter, 1968). Moreover, all the clinical groups investigated by Kelly and Walter as well as the present group of agoraphobic patients show smaller responses to experimental stressors than normal individuals even if the difference is not large. Wilder's 'Law of Initial Value' (1957) is a more precisely formulated view of the limited "capacity to react'. Wilder would argue that the degree of response would be inversely proportional to the initial ('basal') level of blood flow. The present results are, of course, in some agreement with this law in that combining both groups the degree of response correlates $(\rho=$ $-0 \cdot 36$ ) with initial value of blood flow. In addition Wilder (1957) draws attention to the situation where a high initial value becomes associated with a negative response-that is, a fall in blood flow under stress, and indeed three of the six highest initial values show negative stress responses. Certainly further investigation of stress response phenomena should bear the Law of Initial Value in mind.

No investigation was attempted here of the occurrence of symptoms such as depersonalization under the stressed conditions. It could certainly be that, as Roth and Harper (1962) and Lader and Wing (1964) suggested, depersonalization acts as a defensive mechanism reducing the impact of the stressor and protecting the organism against excessive autonomic arousal. An alternative explanation could be that the anxious patient experiences a welter of stressful stimuli in life and is in a continuous state of almost full habituation or inhibition. The biochemical study of Friedman, Mason, and Hamburg (1963) shows a rather similar result in a study of corticosteroid excretion in the parents of children with neoplastic disease. Marked increases in 17hydroxycorticosteroid excretion are commonly observed in subjects under acute psychological stresses. The parents of the children in Friedman's study were exposed to long-term stress after the discovery of the illness, but it was found that even the death of the child increased the corticosteroid level of the parents to only a small degree. In addition it was noted that the formation of rigid defences against anxiety was associated with particularly small stress responses. One might indeed speculate on the possible relationship between experiences such as depersonalization and an inhibition of autonomic reactivity.

General observations suggest that the inhibition of reactivity to stress may be selective and autonomic responses become highly specific to some situations. No simple explanation of how this might arise seems possible however.

This experiment does not provide any simple support for the positive feed-back hypothesis in the production of phobic symptoms. It would appear doubtful if the low habituation rate found here under stress can be regarded as relevant, in that no special stress reaction was demonstrated. Similarly, the possibility of using such a measure for monitoring desensitization in agoraphobic patients remains unclarified, since either specific anxieties were not precipitated by the stressor or not measured by muscle blood flow. If habituating blood flow could be demonstrated in responses to the special stimuli used in desensitization, their utility would then depend upon whether or not it could be shown that anxiety or simply autonomic arousal habituates.

Under relaxation conditions, habituation continued gradually with little evidence of completion within the 30 minute period. This is important in considering methods of measurement, and the empirical nature of 'basal' values as calculated by Kelly's method is emphasized. It is interesting that under conditions of measurement employed by Kelly and Walter (1968) habituation in one group is also apparent in non-stress conditions. It would appear, therefore, that the use of regression constants as indices of blood flow is likely to prove 
more meaningful. They seem much less likely to be invalidated by experimental errors.

\section{CONCLUSIONS}

Forearm muscle blood flow is higher in agoraphobic patients than in normal controls while relaxing. It is hypothesized that this constant difference represents 'free floating anxiety' level.

Habituation over a substantial period of time is demonstrated and the suggestion is made that regression constants are better indices of blood flow than empirical values such as the mean of three lowest measurements.

A lack of mean response to an experimental stressor was found among the phobic group in contrast to a large rapidly habituating response shown by normal subjects. In view of the lack of stress responsiveness of the phobic patients no new light is thrown on the positive feed-back hypothesis.

Comparison of the 'stressed' blood flow responses of normals and phobics is strikingly similar to the pattern of PGR (psychogalvanic reflex) responses.

No simple application of physiological measurements to the assessment of anxiety in therapeutic situations appears likely from this research.

I am grateful to Professor Roth for his interest in this research, and to Dr. Alan Kerr for his help in selecting the group of agoraphobic patients.

\section{REFERENCES}

Barcroft, H., and Edholm, O. G. (1945). On the vasodilation in human skeletal muscle during post-haemorrhagic fainting. J. Physiol. (Lond.), 104, 161-175.

-, and Swan, H. J. C. (1953). The Sympathetic Control of Human Blood Vessels. Arnold, London.

Friedman, S. B., Mason, J. W., and Hamburg, D. A. (1963). Urinary 17-hydroxycorticosteroid levels in parents of children with neoplastic disease. A study of chronic psychological stres $Z_{\mathbb{D}}$ Psychosom. Med., 25, 364-376.

Harper, M., Gurney, C., Savage, R. D., and Roth, M. (1965). Forearn blood flow in normal subjects and patients with phobic anxieten states. Brit. J. Psychiat., 111, 723-731.

Kelly, D. H. W. (1966). Measurement of anxiety by forearm blod flow. Brit. J. Psychiat., 112, 789-798.

_- Walter, C. J. S., and Sargant, W. (1966). Modified leucotome assessed by forearm blood flow and other measurements. Brio J. Psychiat., 112, 871-881.

(1967). The technique of forearm plethysmography for assessing anxiety. J. psychosom. Res., 10, 373-382.

- and Walter, C. J. S. (1968). The relationship between clinicàr diagnosis and anxiety assessed by forearm blood flow and othef measurements. Brit. J. Psychiat., 114, 611-627.

Kerslake, D. McK. (1949). The effect of the application of an arteriat occlusion cuff to the wrist on the blood flow in the human fore arm. J. Physiol. (Lond.), 108, 451-457.

Lader, M. H., and Montagu, J. D. (1962). The psycho-galvanic reflex a pharmacological study of the peripheral mechanism.J. Neuro雨 Neurosurg. Psychiat., 25, 126-133.

- (1964). The effect of cyclobarbitone on the habituation of the psycho-galvanic reflex. Brain, 87, 321-340.

$\longrightarrow$, and Wing, L. (1964). Habituation of the psycho-galvanic refle $\vec{W}$ in patients with anxiety states and in normal subjects. $J$ Neurol. Neurosurg. Psychiat., 27, 210-218.

- - (1965). Comparative bioassay of chlordiazepoxide anfy amylobarbitone sodium therapies in patients with anxiety states, using psychological and clinical measures. J. NeuroI Neurosurg. Psychiat., 28, 414-425.

Marks, I. (To be published). Classification of phobic disorders. Paper read to Royal Medico-Psychological Association, LondonJ 1967.

Mathews, A. M. (To be published). The evaluation of forearm bibo $\vec{d}$ flow as a psychophysiological measure. Paper read to BQistr Psychological Society, London Conference, December 196i⿱一.

Roth, M. (1960). The phobic anxiety-depersonalisation syndome and some general aetiological problems in psychiatri f. Neuropsychiat., 1, 293-306.

- and Harper, M. (1962). Temporal lobe epilepsy and the pho b anxiety depersonalisation syndrome. Pt. II Practical and theoretical considerations. Comprehens. Psychiat., 3, 215-206

Vanderhoof, E., and Clancy, J. (1962). Peripheral blood flow a indicator of emotional reaction. J. appl. Physiol., 17, 67-7

- - (1964). Physiological correlate of therapeutic change. gen. Psychiat., 11, 145-150.

Whitney, R. J. (1953). The measurement of volume changes in humaif limbs. J. Physiol. (Lond.), 121, 1-27.

- (1963). The mercury-in-rubber strain gauge plethysmograp Brit. med. Bull., 19, 105-107.

Wilder, J. (1957). The law of initial value in neurology and psychiat facts and problem. J. nerv. ment. Dis., 125, 73-86. 